PROCEEDINGS OF THE

AMERICAN MATHEMATICAL SOCIETY

Volume 134, Number 2, Pages 455-464

S 0002-9939(05)07994-3

Article electronically published on June 29, 2005

\title{
A DUAL GRAPH CONSTRUCTION FOR HIGHER-RANK GRAPHS, AND $K$-THEORY FOR FINITE 2-GRAPHS
}

\author{
STEPHEN ALLEN, DAVID PASK, AND AIDAN SIMS
}

(Communicated by David R. Larson)

\begin{abstract}
Given a $k$-graph $\Lambda$ and an element $p$ of $\mathbb{N}^{k}$, we define the dual $k$-graph, $p \Lambda$. We show that when $\Lambda$ is row-finite and has no sources, the $C^{*}$-algebras $C^{*}(\Lambda)$ and $C^{*}(p \Lambda)$ coincide. We use this isomorphism to apply Robertson and Steger's results to calculate the $K$-theory of $C^{*}(\Lambda)$ when $\Lambda$ is finite and strongly connected and satisfies the aperiodicity condition.
\end{abstract}

\section{INTRODUCTION}

In 1980, Cuntz and Krieger introduced a class of $C^{*}$-algebras, now called CuntzKrieger algebras, associated to finite $\{0,1\}$-matrices $A[4$. Enomoto and Watatani then showed that these algebras could be regarded as being associated in a natural way to finite directed graphs by regarding $A$ as the vertex adjacency matrix of a finite directed graph $E$ [5]. Generalising this association, Enomoto and Watatini associated $C^{*}$-algebras $C^{*}(E)$ to finite graphs $E$ with no sources 1 ( $E$ has no sources if each vertex of $E$ is the range of at least one edge). Although not every finite directed graph with no sources has a vertex adjacency matrix with entries in $\{0,1\}$, the vertex adjacency matrix of the dual graph $\widehat{E}$ formed by regarding the edges of $E$ as vertices and the paths of length 2 in $E$ as edges does always have entries in $\{0,1\}$, and the Cuntz-Krieger algebras associated to $E$ and to $\widehat{E}$ are canonically isomorphic [11. These results have since been extended to infinite graphs (see for example [10, 9, 3, 7]; see also [2] when $E$ has sources).

One of the major attractions of graph algebras is their applicability to the classification program for simple purely infinite nuclear $C^{*}$-algebras. Conditions on a graph $E$ have been identified which guarantee that $C^{*}(E)$ is purely infinite, simple, and nuclear, and satisfies the Universal Coefficient Theorem (see, for example, 3]), thus producing a large class of directed graphs whose $C^{*}$-algebras are determined up to isomorphism by their $K$-theory [12. The $K$-theory of $C^{*}(E)$ for an arbitrary directed graph $E$ was calculated in [13, and it is shown in 17 that given any two finitely generated abelian groups $G, H$ such that $H$ is torsion free, there exists a

Received by the editors March 22, 2004 and, in revised form, September 20, 2004.

2000 Mathematics Subject Classification. Primary 46L05.

Key words and phrases. Graphs as categories, graph algebra, $C^{*}$-algebra, $K$-theory.

This research was supported by the Australian Research Council.

${ }^{1}$ For the sake of consistency with $k$-graph notation, we regard directed graphs as 1 -graphs, so no sources here corresponds to no sinks in, for example, [5] 3].

(C)2005 American Mathematical Society Reverts to public domain 28 years from publication 
directed graph $E$ such that $C^{*}(E)$ is simple, purely infinite, nuclear, and satisfies the Universal Coefficient Theorem, with $K_{0}\left(C^{*}(E)\right) \cong G$ and $K_{1}\left(C^{*}(E)\right) \cong H$.

In 1999, Robertson and Steger introduced a class of higher-rank Cuntz-Krieger algebras $\mathcal{A}$, associated to collections $M_{1}, \ldots, M_{k}$ of commuting $\{0,1\}$-matrices satisfying appropriate compatibility conditions [15]. In [16], they went on to calculate the $K$-theory of $\mathcal{A}$, demonstrating in particular that $K_{1}(\mathcal{A})$ need not be torsion free, so that the class of higher-rank Cuntz-Krieger algebras exhausts some $K$-invariants which are not achieved by graph algebras. In order to place these higher-rank Cuntz-Krieger algebras in a graph-theoretic setting, and to generalise them as Watatani and Enomoto had generalised the original Cuntz-Krieger algebras, Kumjian and Pask introduced the notion of a higher-rank graph $\Lambda$, and defined and investigated the associated higher-rank graph $C^{*}$-algebra $C^{*}(\Lambda)$ [ . Connectivity in a rank- $k$ graph $\Lambda$ is described in terms of $k$ commuting vertex adjacency matrices $\left\{M_{1}^{\Lambda}, \ldots, M_{k}^{\Lambda}\right\}$, called coordinate matrices. Just as in the rank-1 setting, not every $k$-graph has coordinate matrices with entries in $\{0,1\}$, but if $\Lambda$ is a $k$-graph whose coordinate matrices are $\{0,1\}$-matrices, then [8, Corollary 3.5(ii)] shows that $C^{*}(\Lambda)$ and the $C^{*}$-algebra $\mathcal{A}$ associated to the coordinate matrices as in 15 are identical.

In this paper we introduce a notion of a dual graph for higher-rank graphs, and show that for a large class of higher-rank graphs $\Lambda$, the dual higher-rank graph $p \Lambda$ and the original higher-rank graph $\Lambda$ have canonically isomorphic $C^{*}$-algebras for all $p \in \mathbb{N}^{k}$ (cf. [1]). We also show that by choosing $p$ appropriately, we can ensure that $p \Lambda$ has coordinate matrices with entries in $\{0,1\}$. Using these results, we identify a class of finite rank-2 graphs whose $C^{*}$-algebras are isomorphic to the rank-2 Cuntz-Krieger algebras studied by Robertson and Steger, and we use the results of [16] to show that these $C^{*}$-algebras are purely infinite, simple, unital and nuclear, and to calculate their $K$-theory.

The layout of the paper is as follows: in Section 2, we recall the definition of $k$-graphs and the associated notation; in Section 3 we introduce the dual graph construction for $k$-graphs, and show that this construction preserves the associated $C^{*}$-algebra; and in Section 4 we identify the finite 2 -graphs $\Lambda$ whose $C^{*}$-algebras can be studied using Robertson and Steger's results, and use these results to calculate $K_{*}\left(C^{*}(\Lambda)\right)$.

In the final stages of preparation of this paper, the authors became aware of Evans' Ph.D. thesis [6], which appears to obtain more general results regarding $K$-theory for 2-graph $C^{*}$-algebras than those established here. The authors thank the referee for helpful comments which we feel have improved the exposition.

\section{Preliminaries}

We regard $\mathbb{N}^{k}$ as an additive semigroup with identity 0 . Given $m, n \in \mathbb{N}^{k}$, we write $m \vee n$ for their coordinate-wise maximum and $m \wedge n$ for their coordinate-wise minimum, and if $m \leq n$, then we write $[m, n]$ for the set $\left\{p \in \mathbb{N}^{k}: m \leq p \leq n\right\}$. We denote the canonical generators of $\mathbb{N}^{k}$ by $\left\{e_{1}, \ldots, e_{k}\right\}$, and for $n \in \mathbb{N}^{k}$, we write $n_{j}$ for the $j^{\text {th }}$ coordinate of $n$ ).

Definition 2.1. Let $k \in \mathbb{N} \backslash\{0\}$. A $k$-graph is a pair $(\Lambda, d)$ where $\Lambda$ is a countable category and $d$ is a functor from $\Lambda$ to $\mathbb{N}^{k}$ which satisfies the factorisation property: if $\lambda \in \operatorname{Mor}(\Lambda)$ and $d(\lambda)=m+n$, then there are unique morphisms $\mu \in d^{-1}(m)$ and $\nu \in d^{-1}(n)$ such that $\lambda=\mu \nu$. 
We refer to elements of $\operatorname{Mor}(\Lambda)$ as paths and to elements of $\operatorname{Obj}(\Lambda)$ as vertices and we write $r$ and $s$ for the codomain and domain maps. The factorisation property allows us to identify $\operatorname{Obj}(\Lambda)$ with $\{\lambda \in \operatorname{Mor}(\Lambda): d(\lambda)=0\}$. So we write $\lambda \in \Lambda$ in place of $\lambda \in \operatorname{Mor}(\Lambda)$, and when $d(\lambda)=0$, we regard $\lambda$ as a vertex of $\Lambda$.

Given $\lambda \in \Lambda$ and $E \subset \Lambda$, we define $\lambda E=\{\lambda \mu: \mu \in E, r(\mu)=s(\lambda)\}$ and $E \lambda=\{\mu \lambda: \mu \in E, s(\mu)=r(\lambda)\}$. In particular if $d(v)=0$, then $v$ is a vertex of $\Lambda$ and $v E=\{\lambda \in E: r(\lambda)=v\}$; similarly, $E v=\{\lambda \in \Lambda: s(\lambda)=v\}$. We write $\Lambda^{n}$ for the collection $\{\lambda \in \Lambda: d(\lambda)=n\}$.

Definition 2.2. We say that a $k$-graph $(\Lambda, d)$ is row-finite if $v \Lambda^{n}$ is finite for all $v \in \Lambda^{0}$ and $n \in \mathbb{N}^{k}$, and that $\Lambda$ has no sources if $v \Lambda^{n}$ is nonempty for all $v \in \Lambda^{0}$ and $n \in \mathbb{N}^{k}$. We say that $\Lambda$ is strongly connected if $v \Lambda w$ is nonempty for all $v, w \in \Lambda^{0}$, and we say that $\Lambda$ is finite if $\Lambda^{0}$ and each $\Lambda^{e_{i}}$ are finite.

The factorisation property ensures that if $l \leq m \leq n \in \mathbb{N}^{k}$ and if $d(\lambda)=n$, then there exist unique paths denoted $\lambda(0, l), \lambda(l, m)$ and $\lambda(m, n)$ such that $d(\lambda(0, l))=l$, $d(\lambda(l, m))=m-l$, and $d(\lambda(m, n))=n-m$ and such that $\lambda=\lambda(0, l) \lambda(l, m) \lambda(m, n)$.

Given $k \in \mathbb{N} \backslash\{0\}$, and $k$-graphs $\left(\Lambda_{1}, d_{1}\right)$ and $\left(\Lambda_{2}, d_{2}\right)$, we call a covariant functor $x: \Lambda_{1} \rightarrow \Lambda_{2}$ a graph morphism if it satisfies $d_{2} \circ x=d_{1}$.

Definition 2.3. As in [8], given $k \in \mathbb{N} \backslash\{0\}$, we write $\Omega_{k}$ for the $k$-graph given by $\operatorname{Obj}\left(\Omega_{k}\right)=\mathbb{N}^{k}, \operatorname{Mor}\left(\Omega_{k}\right)=\left\{(m, n) \in \mathbb{N}^{k} \times \mathbb{N}^{k}: m \leq n\right\}, r(m, n)=m, s(m, n)=n$, $(m, n) \circ(n, p)=(m, p)$, and $d(m, n)=n-m$. Given a $k$-graph $\Lambda$, an infinite path of $\Lambda$ is a graph morphism $x: \Omega_{k} \rightarrow \Lambda$. We denote the collection of all infinite paths of $\Lambda$ by $\Lambda^{\infty}$. For $p \in \mathbb{N}^{k}$, we write $\sigma^{p}: \Lambda^{\infty} \rightarrow \Lambda^{\infty}$ for the shift-map determined $\sigma^{p}(x)(m, n)=x(m+p, n+p)$, and we say that $x \in \Lambda^{\infty}$ is aperiodic if there do not exist $p, q \in \mathbb{N}^{k}$ with $p \neq q$ and $\sigma^{p}(x)=\sigma^{q}(x)$.

Definition 2.4. Let $(\Lambda, d)$ be a row-finite $k$-graph with no sources. A CuntzKrieger $\Lambda$-family is a collection $\left\{t_{\lambda}: \lambda \in \Lambda\right\}$ of partial isometries satisfying

(i) $\left\{t_{v}: v \in \Lambda^{0}\right\}$ is a collection of mutually orthogonal projections;

(ii) $t_{\lambda} t_{\mu}=t_{\lambda \mu}$ whenever $s(\lambda)=r(\mu)$;

(iii) $t_{\lambda}^{*} t_{\lambda}=t_{s(\lambda)}$ for all $\lambda \in \Lambda$; and

(iv) $t_{v}=\sum_{\lambda \in v \Lambda^{n}} t_{\lambda} t_{\lambda}^{*}$ for all $v \in \Lambda^{0}$ and $n \in \mathbb{N}^{k}$.

The Cuntz-Krieger algebra $C^{*}(\Lambda)$ is the $C^{*}$-algebra generated by a Cuntz-Krieger $\Lambda$-family $\left\{s_{\lambda}: \lambda \in \Lambda\right\}$ which is universal in the sense that for every Cuntz-Krieger $\Lambda$-family $\left\{t_{\lambda}: \lambda \in \Lambda\right\}$ there is a unique homomorphism $\pi$ of $C^{*}(\Lambda)$ satisfying $\pi\left(s_{\lambda}\right)=t_{\lambda}$ for all $\lambda \in \Lambda$.

\section{DUAL HIGHER-RANK GRAPHS}

In this section we define the higher-rank analog $p \Lambda$ of the dual graph construction for directed graphs.

Definition 3.1. Let $(\Lambda, d)$ be a $k$-graph and let $p \in \mathbb{N}^{k}$. Let $p \Lambda=\{\lambda \in \Lambda$ : $d(\lambda) \geq p\}$. Define range and source maps on $p \Lambda$ by $r_{p}(\lambda)=\lambda(0, p)$, and $s_{p}(\lambda)=$ $\lambda(d(\lambda)-p, d(\lambda))$ for all $\lambda \in p \Lambda$, and define composition by $\lambda \circ_{p} \mu=\lambda \mu(p, d(\mu))=$ $\lambda(0, d(\lambda)-p) \mu$ whenever $s_{p}(\lambda)=r_{p}(\mu)$. Finally, define a degree map $d_{p}$ on $p \Lambda$ by $d_{p}(\lambda)=d(\lambda)-p$ for all $\lambda \in p \Lambda$.

Proposition 3.2. Let $(\Lambda, d)$ be a k-graph, and let $p \in \mathbb{N}^{k}$. Then $\left(p \Lambda, d_{p}\right)$ is a k-graph. 
Proof. It is straightforward to check that $p \Lambda$ is a category with the indicated operations. If $\lambda, \mu \in p \Lambda$ and $s_{p}(\lambda)=r_{p}(\mu)$, then $\lambda \circ_{p} \mu=\lambda \mu(p, d(\mu))$ by definition, so $d_{p}\left(\lambda \circ_{p} \mu\right)=d(\lambda)+d(\mu)-2 p=d_{p}(\lambda)+d_{p}(\mu)$. Therefore, $d_{p}$ is a functor from $p \Lambda$ to $\mathbb{N}^{k}$.

We need to check that the factorisation property holds for $p \Lambda$. Take any $\lambda \in p \Lambda$ and $m, n \in \mathbb{N}^{k}$ with $m+n=d_{p}(\lambda)$, so $d(\lambda)=m+p+n$. By the factorisation property for $\Lambda$ we have $\lambda=\lambda(0, m) \lambda(m, m+p) \lambda(m+p, m+p+n)$. But then $\lambda=(\lambda(0, m) \lambda(m, m+p)) \circ_{p}(\lambda(m, m+p) \lambda(m+p, m+p+n))$ in $p \Lambda$, and $d_{p}(\lambda(0, m) \lambda(m, m+p))=m$ and $d_{p}(\lambda(m, m+p) \lambda(m+p, m+p+n))=n$. This decomposition is unique by the factorisation property for $\Lambda$.

Remark 3.3. If $\Lambda$ has no sources, then $p \Lambda$ has no sources, and if $\Lambda$ is row-finite, then $p \Lambda$ is row-finite.

Proposition 3.4. Let $(\Lambda, d)$ be a k-graph, and let $p, q \in \mathbb{N}^{k}$. Then $q(p \Lambda)=(q+p) \Lambda$.

Proof. By definition, we have $q(p \Lambda)^{n}=p \Lambda^{(n+q)}=\Lambda^{(n+q+p)}=(q+p) \Lambda^{n}$ for all $n \in \mathbb{N}$. Hence $q(p \Lambda)$ and $(q+p) \Lambda$ have identical elements. For the remainder of the proof, we write $s_{q}^{p \Lambda}, r_{q}^{p \Lambda}, \circ_{q}^{p \Lambda}$, and $d_{q}^{p \Lambda}$ for the source, range, composition and degree maps of the dual graph $q(p \Lambda)$.

Fix $\lambda \in \Lambda^{n+p+q}$. We have $s_{(q+p)}(\lambda)=\lambda(n, n+p+q)$ by definition, while $s_{q}^{p \Lambda}(\lambda)$ is the final segment $\mu$ of $\lambda$ such that $d(\mu)-p=d_{p}(\mu)=q$; that is, $d(\mu)=p+q$. Hence $s_{p+q}(\lambda)=s_{q}^{p \Lambda}(\lambda)$. Similarly, $r_{p+q}(\lambda)=\lambda(0, p+q)=r_{q}^{p \Lambda}(\lambda)$. Moreover, $d_{p+q}(\lambda)=d(\lambda)-(p+q)=d_{p}(\lambda)-q=d_{q}^{p \Lambda}(\lambda)$. Since $\lambda$ was arbitrary, it follows that the range, source, and degree maps for $(p+q) \Lambda$ and $q(p \Lambda)$ agree.

This having been established, we have $r_{p+q}(\lambda)=s_{p+q}(\mu)$ if and only if $r_{q}^{p \Lambda}(\lambda)=$ $s_{q}^{p \Lambda}(\mu)$, in which case both $\lambda \circ_{p+q} \mu$ and $\lambda \circ_{q}^{p \Lambda} \mu$ are equal to $\lambda \mu(p+q, d(\mu))$ by definition, completing the proof.

Theorem 3.5. Let $(\Lambda, d)$ be a row-finite $k$-graph with no sources, and let $p \in \mathbb{N}^{k}$. Let $\left\{s_{\lambda}: \lambda \in \Lambda\right\}$ denote the universal generating Cuntz-Krieger $\Lambda$-family in $C^{*}(\Lambda)$, and let $\left\{t_{\lambda}: \lambda \in \Lambda\right\}$ be the universal generating Cuntz-Krieger $p \Lambda$-family in $C^{*}(p \Lambda)$. For all $\lambda \in p \Lambda$, define $r_{\lambda}=s_{\lambda} s_{s_{p}(\lambda)}^{*}$. There is an isomorphism $\phi: C^{*}(p \Lambda) \rightarrow C^{*}(\Lambda)$ such that $\phi\left(t_{\lambda}\right)=r_{\lambda}$ for all $\lambda \in p \Lambda$.

Proof. First we show that the family $\left\{r_{\lambda}: \lambda \in p \Lambda\right\}$ is a Cuntz-Krieger $p \Lambda$-family. Since, for any $\beta \in p \Lambda^{0}$, we have $s_{\beta} \neq 0$, it follows that $r_{\beta}=s_{\beta} s_{\beta}^{*} \neq 0$ and that it is a projection in $C^{*}(\Lambda)$. Furthermore, for distinct $\alpha, \beta \in p \Lambda^{0}$, we have

$$
r_{\alpha} r_{\beta}=s_{\alpha} s_{\alpha}^{*} s_{\beta} s_{\beta}^{*}=\delta_{\alpha, \beta} s_{\alpha} s_{\beta}^{*}=\delta_{\alpha, \beta} r_{\alpha} .
$$

This establishes relation (i).

For relation (ii), let $\mu, \nu \in p \Lambda$ with $r_{p}(\nu)=s_{p}(\mu)$, so $\mu \circ_{p} \nu=\mu \nu(p, d(\nu))$. Then,

$$
r_{\mu \circ_{p} \nu}=s_{\mu \circ_{p} \nu} s_{s_{p}\left(\mu \circ_{p} \nu\right)}^{*}=s_{\mu} s_{\nu(p, d(\nu))} s_{s_{p}(\nu)}^{*}=s_{\mu} s_{s_{p}(\mu)}^{*} s_{s_{p}(\mu)} s_{\nu(p, d(\nu))} s_{s_{p}(\nu)}^{*} .
$$

But $s_{p}(\mu)=r_{p}(\nu)=\nu(0, p)$, so we can rewrite the right-hand side of (3.1) to obtain $r_{\mu \circ_{p} \nu}=s_{\mu} s_{s_{p}(\mu)}^{*} s_{\nu} s_{s_{p}(\nu)}^{*}=r_{\mu} r_{\nu}$. This establishes relation (ii).

Let $\lambda \in p \Lambda$, say $d_{p}(\lambda)=n$. Then $r_{\lambda}^{*} r_{\lambda}=s_{s_{p}(\lambda)} s_{\lambda}^{*} s_{\lambda} s_{s_{p}(\lambda)}^{*}=s_{s_{p}(\lambda)} s_{s_{p}(\lambda)}^{*}=r_{s_{p}(\lambda)}$ by definition, establishing relation (iii).

Finally, for relation (iv), let $\beta \in p \Lambda^{0}$ and let $n \in \mathbb{N}^{k}$. Then

$$
r_{\beta}=s_{\beta} s_{\beta}^{*}=\sum_{\gamma \in s(\beta) \Lambda^{n}} s_{\beta} s_{\gamma} s_{\gamma}^{*} s_{\beta}^{*}=\sum_{\lambda \in \beta \Lambda^{n}} s_{\lambda} s_{\lambda}^{*} .
$$


Applying the factorisation property and relation (ii) for $C^{*}(\Lambda)$ to the right-hand side then gives

$$
r_{\beta}=\sum_{\lambda \in \beta \Lambda^{n}} s_{\lambda(0, n)} s_{\lambda(n, n+p)} s_{\lambda(n, n+p)}^{*} s_{\lambda(0, n)}^{*},
$$

and then since each $s_{\lambda(n, n+p)} s_{\lambda(n, n+p)}^{*}$ is a projection, we obtain

$$
r_{\beta}=\sum_{\lambda \in \beta \Lambda^{n}}\left(s_{\lambda(0, n)} s_{\lambda(n, n+p)} s_{\lambda(n, n+p)}^{*}\right)\left(s_{\lambda(n, n+p)} s_{\lambda(n, n+p)}^{*} s_{\lambda(0, n)}^{*}\right)=\sum_{\lambda \in \beta\left(p \Lambda^{n}\right)} r_{\lambda} r_{\lambda}^{*},
$$

which establishes relation (iv).

It follows from the universal property of $C^{*}(p \Lambda)$ that there exists a homomorphism $\phi: C^{*}(p \Lambda) \rightarrow C^{*}(\Lambda)$ satisfying $\phi\left(t_{\lambda}\right)=r_{\lambda}$ for all $\lambda \in p \Lambda$. We claim that $\left\{r_{\lambda}: \lambda \in p \Lambda\right\}$ generates $C^{*}(\Lambda)$. To see this, let $\sigma \in \Lambda$ with $d(\sigma)=n$. An application of relation (iv) for $C^{*}(\Lambda)$ gives $s_{\sigma}=\sum_{\beta \in s(\sigma) \Lambda^{p}} s_{\sigma} s_{\beta} s_{\beta}^{*}=\sum_{\lambda \in \sigma \Lambda^{p}} s_{\lambda} s_{s_{p}(\lambda)}^{*}$, and this last is equal to $\sum_{\lambda \in \sigma \Lambda^{p}} r_{\lambda}$ by definition. Thus $\phi$ maps $C^{*}(p \Lambda)$ onto $C^{*}(\Lambda)$.

Now let $\gamma^{\Lambda}$ denote the gauge action on $C^{*}(\Lambda)$, and let $\gamma^{p \Lambda}$ denote the gauge action on $C^{*}(p \Lambda)$. For $z \in \mathbb{T}^{k}$ and $\lambda \in p \Lambda$, we have

$$
\gamma_{z}^{\Lambda}\left(r_{\lambda}\right)=\gamma_{z}^{\Lambda}\left(s_{\lambda} s_{s_{p}(\lambda)}^{*}\right)=z^{d(\lambda)} s_{\lambda}\left(z^{d\left(s_{p}(\lambda)\right)} s_{s_{p}(\lambda)}\right)^{*}=z^{d(\lambda)-p} r_{\lambda}=\gamma^{p \Lambda}\left(r_{\lambda}\right) .
$$

Theorem 3.4 of $[8$ now establishes that $\phi$ is injective.

Remark 3.6. The hypotheses that $\Lambda$ be row-finite and have no sources are crucial in Theorem 3.5. To see why, note that for $v \in \Lambda^{0}$, the generator $s_{v}$ of $C^{*}(\Lambda)$ is recovered in $C^{*}(p \Lambda)$ as $\sum_{\beta \in p \Lambda^{0}, r(\beta)=v} r_{\beta}$. However, even for 1-graphs the Cuntz-Krieger relations only insist that $p_{v}=\sum_{r(e)=v} s_{e} s_{e}^{*}$ when $r^{-1}(v)$ is finite and nonempty.

Lemma 3.7. Let $(\Lambda, d)$ be a $k$-graph, and let $p \in \mathbb{N}^{k}$. For each $n \in \mathbb{N}^{k}$ with $n \leq p$ and $v, w \in p \Lambda^{0}$, there is at most one $\lambda \in v\left(p \Lambda^{n}\right) w$.

Proof. Let $v, w \in p \Lambda^{0}=\Lambda^{p}$ and suppose $\lambda \in v\left(p \Lambda^{n}\right) w$. Then $\lambda \in \Lambda^{n+p}, \lambda(0, p)=v$, and $\lambda(n, n+p)=w$. Since $n \leq p$ we have $\lambda(0, n)=(\lambda(0, p))(0, n)=v(0, n)$, so $\lambda=\lambda(0, n) \lambda(n, n+p)=v(0, n) w$, and hence is determined by $v$ and $w$.

Notation 3.8. Let $(\Lambda, d)$ be a $k$-graph. We write $M_{i}^{\Lambda}, 1 \leq i \leq k$, for the matrices in $M_{\Lambda^{0}}(\mathbb{N})$ defined by $\left(M_{i}^{\Lambda}\right)_{v, w}=\left|w \Lambda^{e_{i}} v\right|$ for $w, v \in \Lambda^{0}$, and we refer to these matrices as the coordinate matrices of $\Lambda$.

Remark 3.9. In [8, 6] $\left(M_{i}^{\Lambda}\right)_{v, w}=\left|v \Lambda_{e_{i}} w\right|$, so our $M_{i}^{\Lambda}$ is the transpose of theirs. This is for consistency with the matrices in [16, 15]; we will be employing Robertson and Steger's results to calculate $K$-theory in Section 4 .

Corollary 3.10. Let $(\Lambda, d)$ be a k-graph, and let and $p \in \mathbb{N}^{k}$ with $p_{i} \geq 1$ for $1 \leq i \leq k$. Then the coordinate matrices $M_{i}^{p \Lambda}$ of $p \Lambda$ are $\{0,1\}$-matrices.

\section{4. $K$-THEORY}

In this section we identify a class of 2 -graphs whose associated $C^{*}$-algebras are isomorphic to higher rank Cuntz-Krieger algebras in the sense of [16, and use the results of [16] to calculate the $K$-theory of the $C^{*}$-algebras of such 2-graphs. To state the main theorem for this section we employ the following notation: given square $n \times n$ matrices $M, N$, we write $\left[\begin{array}{ll}M & N\end{array}\right]$ for the block $n \times 2 n$ matrix whose first $n$ columns are those of $M$ and whose last $n$ columns are those of $N$. We also write $\mathbf{1}$ for the element $(1,1)$ of $\mathbb{N}^{2}$. 
Theorem 4.1. Let $(\Lambda, d)$ be a 2-graph which is finite and strongly connected as in Definition 2.2 and which has an aperiodic infinite path as in Definition 2.3. Then $C^{*}(\Lambda)$ is purely infinite, simple, unital and nuclear, and we have

$$
\begin{aligned}
& \operatorname{rank}\left(K_{0}\left(C^{*}(\Lambda)\right)\right)=\operatorname{rank}\left(K_{1}\left(C^{*}(\Lambda)\right)\right) \\
& =\operatorname{rank}\left(\operatorname{coker}\left[\begin{array}{ll}
I-M_{1}^{1 \Lambda} & I-M_{2}^{1 \Lambda}
\end{array}\right]\right) \\
& +\operatorname{rank}\left(\operatorname{coker}\left[I-\left(M_{1}^{1 \Lambda}\right)^{t} \quad I-\left(M_{2}^{1 \Lambda}\right)^{t}\right]\right) \text {; } \\
& \operatorname{tor}\left(K_{0}\left(C^{*}(\Lambda)\right)\right) \cong \operatorname{tor}\left(\operatorname{coker}\left[I-M_{1}^{1 \Lambda} \quad I-M_{2}^{1 \Lambda}\right]\right) ; \text { and } \\
& \operatorname{tor}\left(K_{1}\left(C^{*}(\Lambda)\right)\right) \cong \operatorname{tor}\left(\operatorname{coker}\left[I-\left(M_{1}^{1 \Lambda}\right)^{t} \quad I-\left(M_{2}^{1 \Lambda}\right)^{t}\right]\right) \text {. }
\end{aligned}
$$

The remainder of this section constitutes the proof of Theorem 4.1. We begin by recalling some definitions from [16. Let $A$ be a finite set, and let $M_{1}, M_{2}$ be $A \times A$ matrices with entries in $\{0,1\}$. For $n \in \mathbb{N}^{k}$, let $W_{n}=\{w:[0, n] \rightarrow A$ : $M_{j}\left(w\left(l+e_{j}\right), w(l)\right)=1$ whenever $\left.l, l+e_{j} \in[0, n]\right\}$; we refer to the elements of $W_{n}$ as allowable words of shape $n$, and write $W$ for the collection $\bigcup_{n \in \mathbb{N}^{2}} W_{n}$ of all allowable words. For $u \in W$, write $S(u)$ for the shape of $u$; that is, $S(u)$ is the unique element of $\mathbb{N}^{2}$ such that $u \in W_{S(u)}$. We identify $W_{0}$ with $A$. The matrices $M_{1}, M_{2}$ are said to satisfy (H0)-(H3) if

(H0) Each $M_{i}$ is nonzero;

(H1a) $M_{1} M_{2}=M_{2} M_{1}$

(H1b) $M_{1} M_{2}$ is a $\{0,1\}$-matrix;

(H2) the directed graph with a vertex for each $a \in A$ and a directed edge $(a, i, b)$ from $a$ to $b$ for each $a, i, b$ such that $M_{i}(b, a)=1$ is irreducible; and

(H3) for each $m \in \mathbb{Z}^{2} \backslash\{0\}$, there exists a word $w \in W$ and elements $l_{1}, l_{2}$ of $\mathbb{N}^{2}$ with $0 \leq l_{1}, l_{2} \leq S(w)$ such that $l_{2}-l_{1}=m$ and $w\left(l_{1}\right) \neq w\left(l_{2}\right)$.

Notation 4.2. If $(\Lambda, d)$ is a 2-graph such that the coordinate matrices $M_{1}^{\Lambda}$ and $M_{2}^{\Lambda}$ are $\{0,1\}$-matrices, we write $W_{n}^{\Lambda}$ and $W^{\Lambda}$ for the collection of allowable words of shape $n$ and for the collection of all allowable words, respectively. For $\lambda \in \Lambda$, let $w_{\lambda}^{\Lambda}$ be the word in $W_{d(\lambda)}^{\Lambda}$ given by $w_{\lambda}^{\Lambda}(m)=s(\lambda(0, m))$ for $0 \leq m \leq d(\lambda)$. Since each $M_{i}^{\Lambda}$ is a $\{0,1\}$-matrix, the map $\lambda \mapsto w_{\lambda}^{\Lambda}$ is a bijection between $\Lambda^{n}$ and $W_{n}^{\Lambda}$ for all $n \in \mathbb{N}^{2}$.

Proposition 4.3. Let $(\Lambda, d)$ be a finite 2-graph with no sources, and let $M_{1}^{\mathbf{1 \Lambda}}$ and $M_{2}^{1 \Lambda}$ be the matrices associated to $\mathbf{1} \Lambda$. Then

(1) $M_{1}^{1 \Lambda}, M_{2}^{1 \Lambda}$ satisfy (H0), (H1a), and (H1b);

(2) $M_{1}^{1 \Lambda}, M_{2}^{1 \Lambda}$ satisfy (H2) if and only if $\Lambda$ is strongly connected; and

(3) if $M_{1}^{1 \Lambda}, M_{2}^{1 \Lambda}$ satisfy (H2), then they satisfy (H3) if and only if $\Lambda$ has an aperiodic infinite path.

Proof. For (1), note that each $M_{i}^{1 \Lambda}$ is a finite square matrix over $1 \Lambda^{0}$ by definition, and has entries in $\{0,1\}$ by Corollary 3.10 . It is easy to see that

$$
\left(M_{i}^{\mathbf{1} \Lambda} M_{3-i}^{1 \Lambda}\right)_{v, w}=\left|\left\{(\alpha, \beta) \in w\left(\mathbf{1} \Lambda^{e_{3-i}}\right) \times\left(\mathbf{1} \Lambda^{e_{i}}\right) v: r(\alpha)=s(\beta)\right\}\right|=\left|w\left(\mathbf{1} \Lambda^{\mathbf{1}}\right) v\right|
$$

for $i=1,2$, which establishes (H1a) and, when combined with Lemma 3.7, (H1b). 
For (2), notice that $M_{1}^{\mathbf{1} \Lambda}, M_{2}^{\mathbf{1} \Lambda}$ satisfy (H2) if and only if for every $v, w \in \mathbf{1} \Lambda^{0}$ there exist elements $\alpha_{1}, \ldots, \alpha_{k}$ in $\mathbf{1} \Lambda^{(1,0)} \cup \mathbf{1} \Lambda^{(0,1)}$ such that $r\left(\alpha_{1}\right)=v, s\left(\alpha_{k}\right)=w$, and $r\left(\alpha_{i+1}\right)=s\left(\alpha_{i}\right)$ for $1 \leq i \leq k-1$.

So suppose first that $\bar{M}_{1}^{\mathbf{1} \Lambda}, M_{2}^{1 \Lambda}$ satisfy (H2), and let $v, w \in \Lambda^{0}$. Since $\Lambda$ has no sources, there exist $\mu, \nu \in \Lambda^{\mathbf{1}}$ with $r(\mu)=v$ and $r(\nu)=w$; so $\mu, \nu \in \mathbf{1} \Lambda^{0}$ by definition, and (H2) ensures that there is a path $\alpha_{1} \ldots \alpha_{k}$ in $\mathbf{1} \Lambda^{(1,0)} \cup \mathbf{1} \Lambda^{(0,1)}$ with $r_{\mathbf{1}}\left(\alpha_{1}\right)=\mu$ and $s_{\mathbf{1}}\left(\alpha_{k}\right)=\nu$. By definition of $\mathbf{1} \Lambda$, the path $\alpha_{1} \ldots \alpha_{k}$ in $\mathbf{1} \Lambda$ is a path $\lambda \in \Lambda$ with $d(\lambda)=d_{\mathbf{1}}\left(\alpha_{1} \ldots \alpha_{k}\right)+\mathbf{1}$, and such that $\lambda(0, \mathbf{1})=\mu$ and $\lambda(d(\lambda)-\mathbf{1}, d(\lambda))=\nu$. But then $\lambda(0, d(\lambda)-\mathbf{1}) \in v \Lambda w$. However, $v, w \in \Lambda^{0}$ were arbitrary, so $\Lambda$ is strongly connected.

Now suppose that $\Lambda$ is strongly connected, and fix $\mu, \nu \in \mathbf{1} \Lambda^{0}$. Since $\Lambda$ is strongly connected, there is a path $\lambda \in s(\mu) \Lambda r(\nu)$, and then $\tau:=\mu \lambda \nu$ belongs to $\mu(\mathbf{1} \Lambda) \nu$ with $d_{\mathbf{1}}(\mu \lambda \nu)=d(\lambda)+\mathbf{1}$. Any factorisation of $\tau$ into segments from $\mathbf{1} \Lambda^{(1,0)} \cup \mathbf{1} \Lambda^{(0,1)}$ now gives a path in $\mathbf{1} \Lambda^{(1,0)} \cup \mathbf{1} \Lambda^{(0,1)}$ from $\nu$ to $\mu$, so $M_{1}^{\mathbf{1 \Lambda}}, M_{2}^{\mathbf{1} \Lambda}$ satisfy (H2).

Finally, for (3), assume that $M_{1}^{1 \Lambda}, M_{2}^{1 \Lambda}$ satisfy (H2), so $\Lambda$ is strongly connected by part (2). For $x \in \Lambda^{\infty}$, define $\mathbf{1} x \in \mathbf{1} \Lambda^{\infty}$ by $(\mathbf{1} x)(m, n)=x(m, n+\mathbf{1})$. It is easy to see that the map $x \mapsto \mathbf{1} x$ is a bijection between $\Lambda^{\infty}$ and $\mathbf{1} \Lambda^{\infty}$.

Claim: $x \in \Lambda^{\infty}$ is aperiodic if and only if $1 x \in \mathbf{1} \Lambda^{\infty}$ is aperiodic. To see this, let $m, n \in \mathbb{N}^{k}$, and fix $x \in \Lambda^{\infty}$. By definition, we have

$$
\begin{aligned}
\sigma^{m}(\mathbf{1} x)=\sigma^{n}(\mathbf{1} x) & \Longleftrightarrow(\mathbf{1} x)(s+m, t+m)=(\mathbf{1} x)(s+n, t+n) \quad \text { for } s \leq t \\
& \Longleftrightarrow x(s+m, t+m+\mathbf{1})=x(s+n, t+n+\mathbf{1}) \quad \text { for } s \leq t .
\end{aligned}
$$

Now if $x(s+m, t+m+\mathbf{1})=x(s+n, t+n+\mathbf{1})$ for all $s \leq t \in \mathbb{N}^{2}$, then the uniqueness of factorisations in $\Lambda$ ensures that $x(s+m, t+m)=x(s+n, t+n)$ for all $s \leq t \in \mathbb{N}^{2}$. Conversely if $x(s+m, t+m)=x(s+n, t+n)$ for all $s \leq t \in \mathbb{N}^{2}$, then replacing $t$ with $t+\mathbf{1}$ gives $x(s+m, t+m+\mathbf{1})=x(s+n, t+n+\mathbf{1})$ for all $s \leq t \in \mathbb{N}^{2}$. Hence (4.4) shows that

$$
\begin{aligned}
\sigma^{m}(\mathbf{1} x)=\sigma^{n}(\mathbf{1} x) & \Longleftrightarrow x(s+m, t+m)=x(s+n, t+n) \quad \text { for } s \leq t \in \mathbb{N}^{2} \\
& \Longleftrightarrow \sigma^{m}(x)=\sigma^{n}(x),
\end{aligned}
$$

establishing the claim. Thus it suffices to show that $M_{i}^{\mathbf{1} \Lambda}$ satisfy (H3) if and only if $\mathbf{1} \Lambda^{\infty}$ has an aperiodic element.

Suppose first that there exists an aperiodic path $x \in \mathbf{1} \Lambda^{\infty}$. Fix $m \in \mathbb{Z}^{2}$, and write $m=m_{+}-m_{-}$where $m_{+}, m_{-} \in \mathbb{N}^{2}$. Since $\left|v\left(\mathbf{1} \Lambda^{e_{i}}\right) w\right| \in\{0,1\}$ for all $v, w \in \mathbf{1} \Lambda^{0}, i=1,2$, we have that $x$ is completely determined by its restriction to the objects of $\Omega_{2}$; that is, by the function from $\mathbb{N}^{2}$ to $\mathbf{1} \Lambda^{0}$ given by $n \mapsto x(n)$. Since $x$ is aperiodic, it follows that $\sigma^{m_{+}}(x)(n) \neq \sigma^{m_{-}}(x)(n)$ for some $n \in \mathbb{N}^{2}$. But then with $N:=n+m_{-}$, we have $x\left(N+m_{+}-m_{-}\right) \neq x(N)$, and $w:=$ $\left.x\right|_{\left[0, N+m_{+}-m_{-}\right]} \in W_{N+m_{+}-m_{-}}^{\mathbf{1 \Lambda}}$ satisfies $w(N) \neq w(N+m)$. Since $m \in \mathbb{Z}^{2}$ was arbitrary, this establishes that $M_{1}^{\mathbf{1} \Lambda}, M_{2}^{\mathbf{1} \Lambda}$ satisfy (H3).

Now suppose that $M_{1}^{\mathbf{1} \Lambda}, M_{2}^{\mathbf{1} \Lambda}$ satisfy (H3). For each $m \in \mathbb{Z}^{2} \backslash\{0\}$, fix $w_{m} \in W^{\mathbf{1} \Lambda}$ and $l_{m} \in \mathbb{N}^{2}$ such that $0 \leq l_{m}, l_{m}+m \leq S\left(w_{m}\right)$ and $w_{m}\left(l_{m}\right) \neq w_{m}\left(l_{m}+m\right)$. Let $\lambda_{m}$ be the unique path in $\mathbf{1} \Lambda$ such that $w_{m}=w_{\lambda_{m}}^{1 \Lambda}$. We will construct an infinite path $x$ which contains infinitely many occurrences of each $\lambda_{m}$; this will ensure that there is no $m$ for which a sufficiently large shift of $x$ has period $m$, and hence that $x$ is aperiodic. The details of this construction, and the verification that the resulting $x$ is aperiodic constitute the remainder of the proof. 
Let $\left\{m_{i}: i \in \mathbb{N}\right\}$ be a listing of $\mathbb{Z}^{2} \backslash\{0\}$. Fix an arbitrary $v \in \mathbf{1} \Lambda^{0}$, and for each $i \in \mathbb{N}$, let $\alpha_{i}$ be any element of $v(\mathbf{1} \Lambda) r\left(\lambda_{m_{i}}\right)$, and let $\beta_{i}$ be any element of $s\left(\lambda_{m_{i}}\right)(\mathbf{1} \Lambda) v$ with the property that $d_{\mathbf{1}}\left(\alpha_{i} \lambda_{m_{i}} \beta_{i}\right) \geq \mathbf{1}$; this is possible because $\Lambda$ is strongly connected and has no sources.

For $i \in \mathbb{N}$, let $\rho_{i}=\alpha_{i} \lambda_{m_{i}} \beta_{i}$, and let $\tau_{i}=\rho_{1} \rho_{2} \ldots \rho_{i}$. Let $x$ be the infinite path $x=\tau_{1} \tau_{2} \tau_{3} \ldots$. We claim that $x$ is aperiodic.

To see this, let $s, t \in \mathbb{N}^{2}$ be distinct, and let $I_{s, t}$ be the element of $\mathbb{N}$ such that $m_{I_{s, t}}=t-s$. Let $J=\max \left\{s_{1}, s_{2}, t_{1}, t_{2}\right\}$; since $d_{\mathbf{1}}\left(\rho_{i}\right) \geq(1,1)$, we have that $i \geq J$ implies $d_{1}\left(\tau_{1} \ldots \tau_{i}\right) \geq s, t$. Let $K=\max \left\{I_{s, t}, J+1\right\}$, and define $N=d_{\mathbf{1}}\left(\tau_{1} \ldots \tau_{K-1}\right)+d_{\mathbf{1}}\left(\rho_{1} \ldots \rho_{I_{s, t}-1}\right)+d\left(\alpha_{I_{s, t}}\right)+l_{t-s}-s$. We have $N \geq 0$ by choice of $K$, and

$$
\begin{aligned}
\sigma^{s}(x)(N) & =x(N+s) \\
& =x\left(d_{\mathbf{1}}\left(\tau_{1} \ldots \tau_{K-1}\right)+d_{\mathbf{1}}\left(\rho_{1} \ldots \rho_{I_{s, t}-1}\right)+d\left(\alpha_{I_{s, t}}\right)+l_{t-s}\right) \\
& =\lambda_{m_{I_{s, t}}}\left(l_{t-s}\right) .
\end{aligned}
$$

A similar calculation shows that $\sigma^{t}(x)(N)=\lambda_{m_{I_{s, t}}}\left(l_{t-s}+(t-s)\right)$, and hence $\sigma^{s}(x)(N) \neq \sigma^{t}(x)(N)$ by our chice of $\lambda_{m_{I_{s, t}}}$. It follows that $\sigma^{s}(x) \neq \sigma^{t}(x)$, and since $s, t \in \mathbb{N}^{2}$ were arbitrary, that $x$ is aperiodic.

Remark 4.4. The preceding proof actually shows: (1) that Proposition 4.3 applies to $M_{i}^{\Lambda}$ for any $\Lambda$ satisfying the property of Lemma 3.7 for $p=\mathbf{1}$; and (2) that $\mathbf{1} \Lambda$ is strongly-connected (resp. strongly connected and contains an infinite path) if and only if $\Lambda$ has the same property. Since our motivation is to prove Theorem 4.1, we have compressed this into a single result.

Notation 4.5. Let $\Lambda$ be a finite strongly connected 2 -graph with an aperiodic infinite path. We write $\mathcal{A}^{\mathbf{1 \Lambda}}$ for the $C^{*}$-algebra associated to $M_{i}^{\mathbf{1 \Lambda}}$ as in [16]. That is, $\mathcal{A}^{\mathbf{1 \Lambda}}$ is the universal $C^{*}$-algebra generated by a family $\left\{s_{u, v}: u, v \in W^{\mathbf{1} \Lambda}, u(S(u))=\right.$ $v(S(v))\}$ of partial isometries satisfying

$$
\begin{aligned}
s_{u, v} & =s_{v, u}^{*} \quad \text { for } u, v \in W^{\mathbf{1 \Lambda}} ; \\
s_{u, v} s_{v, w} & =s_{u, w} \quad \text { for } u, v, w \in W^{\mathbf{1 \Lambda}} ; \\
s_{u, v} & =\sum_{w \in W_{e_{j}}^{\mathbf{1 \Lambda}}, u(S(u))=w(0)} s_{u w} s_{v w}^{*} \text { for } u, v, \in W^{\mathbf{1 \Lambda}}, j \in\{1,2\} ; \text { and } \\
s_{a, a} s_{b, b} & =0 \quad \text { for distinct } a, b \in W_{0}^{\mathbf{1 \Lambda}} .
\end{aligned}
$$

Lemma 4.6. Let $(\Lambda, d)$ be a finite strongly-connected 2-graph which has an aperiodic infinite path. Then $C^{*}(\Lambda)$ is isomorphic to $\mathcal{A}^{\mathbf{1 \Lambda}}$.

Proof. The factorisation property ensures that if $\Lambda$ is strongly connected and contains an infinite path, then $\Lambda$ has no sources. By Theorem 3.5, we have that $C^{*}(\Lambda)$ is isomorphic to $C^{*}(\mathbf{1} \Lambda)$, so it suffices to show that $C^{*}(\mathbf{1} \Lambda)$ is isomorphic to $\mathcal{A}^{\mathbf{1} \Lambda}$. It is easy to check using Definition 2.4(i)-(iv), relations (4.5)-(4.8), and the universal properties of $\mathcal{A}^{\mathbf{1} \Lambda}$ and $C^{*}(\mathbf{1} \Lambda)$ that there exists a homomorphism $\pi: \mathcal{A}^{\mathbf{1} \Lambda} \rightarrow C^{*}(\mathbf{1} \Lambda)$ satisfying $\pi\left(s_{w_{\lambda}^{1 \Lambda}, w_{\mu}^{1 \Lambda}}\right)=s_{\lambda} s_{\mu}^{*}$ for all $\lambda, \mu \in \mathbf{1} \Lambda$, and that there exists a homomorphism $\psi: C^{*}(\mathbf{1} \Lambda) \rightarrow \mathcal{A}^{1 \Lambda}$ satisfying $\psi\left(s_{\lambda}\right)=s_{w_{\lambda}^{1 \Lambda}, w_{s(\lambda)}^{1 \Lambda}}$. Since these two homomorphisms are mutually inverse, the result follows. 
Remark 4.7. The argument of statement (2) of Proposition 4.3 shows that if $\Lambda$ has no sources, then for any $q \geq \mathbf{1}$, the coordinate matrices of $q \Lambda$ will satisfy (H2) only if $\Lambda$ is strongly connected and has no sources. In particular, there exists $q \in \mathbb{N}^{2}$ such that $M_{i}^{q \Lambda}$ satisfy (H0)-(H3) if and only if $M_{i}^{1 \Lambda}$ satisfy (H0)-(H3).

Proof of Theorem 4.1. Theorem 5.9, Proposition 5.11, and Corollary 6.4 of 15, combined with the previous two results, show that $C^{*}(\Lambda)$ is simple, purely infinite and nuclear. We have that $C^{*}(\Lambda)$ is unital with $1_{C^{*}(\Lambda)}=\sum_{v \in \Lambda^{0}} s_{v}$. Proposition 2.14 of [16] establishes (4.1)-(4.3).

Remarks 4.8. (1) The proof of [16, Proposition 2.14] does not make any use of relations (H2) and (H3). Hence the formulas for $K_{*}\left(C^{*}(\Lambda)\right)$ in Theorem 4.1 hold when $\Lambda$ is a finite $k$-graph with no sinks or sources, even if it is not strongly connected and does not have an aperiodic infinite path. However, in this case $C^{*}(\Lambda)$ is not necessarily simple and purely infinite, and so is not determined up to isomorphism by its $K$-theory.

(2) The formulas for $K_{*}\left(C^{*}(\Lambda)\right)$ given in Theorem 4.1 are in terms of the coordinate matrices $M_{i}^{1 \Lambda}$ of the dual $k$-graph. Proposition 5.1 of [6] shows that the same formulas hold if all instances $M_{i}^{1 \Lambda}$ are replaced with $M_{i}^{\Lambda}$, but it is unclear how to show this directly.

\section{REFERENCES}

[1] T. Bates, Applications of the gauge-invariant uniqueness theorem for graph algebras, Bull. Austral. Math. Soc. 65 (2002), 55-67. MR.1922607 (2003g:46064)

[2] T. Bates and D. Pask, Flow equivalence of graph algebras, Ergodic Theory Dynam. Systems 24 (2004), 367-382. MR2054048 (2004m:37019)

[3] T. Bates, D. Pask, I. Raeburn, and W. Szymański, The $C^{*}$-algebras of row-finite graphs, New York J. Math. 6 (2000), 307-324. MR.1777234 (2001k:46084)

[4] J. Cuntz and W. Krieger, A class of $C^{*}$-algebras and topological Markov chains, Invent. Math. 56 (1980), 251-268. MR0561974 (82f:46073a)

[5] M. Enomoto and Y. Watatani, A graph theory for $C^{*}$-algebras, Math. Japon. 25 (1980), 435-442. MR0594544 (83d:46069a)

[6] D. G. Evans, On higher-rank graph $C^{*}$-algebras, Ph.D. Thesis, Univ. Wales, 2002.

[7] N. J. Fowler, M. Laca, and I. Raeburn, The $C^{*}$-algebras of infinite graphs, Proc. Amer. Math. Soc. 128 (2000), 2319-2327. MR.1670363 (2000k:46079)

[8] A. Kumjian and D. Pask, Higher rank graph $C^{*}$-algebras, New York J. Math. 6 (2000) 1-20. MR.1745529 (2001b:46102)

[9] A. Kumjian, D. Pask, and I. Raeburn, Cuntz-Krieger algebras of directed graphs, Pacific J. Math. 184 (1998), 161-174. MR.1626528 (99i:46049)

[10] A. Kumjian, D. Pask, I. Raeburn, and J. Renault, Graphs, groupoids and Cuntz-Krieger algebras, J. Funct. Anal. 144 (1997), 505-541. MR1432596 (98g:46083)

[11] M.H. Mann, I. Raeburn, and C.E. Sutherland, Representations of finite groups and CuntzKrieger algebras, Bull. Austral. Math. Soc. 46 (1992), 225-243. MR1183780 (93k:46046)

[12] N.C. Phillips, A classification theorem for nuclear purely infinite simple $C^{*}$-algebras, Documenta Math. 5 (2000), 49-114. MR.1745197(2001d:46086b)

[13] I. Raeburn and W. Szymański, Cuntz-Krieger algebras of infinite graphs and matrices, Trans. Amer. Math. Soc. 356 (2004), 39-59. MR2020023 (2004i:46087)

[14] I. Raeburn, A. Sims, and T. Yeend, Higher-rank graphs and their $C^{*}$-algebras, Proc. Edinb. Math. Soc 46 (2003), 99-115. MR.1961175 (2004f:46068)

[15] G. Robertson and T. Steger, Affine buildings, tiling systems and higher rank Cuntz-Krieger algebras, J. Reine Angew. Math. 513 (1999), 115-144. MR1713322 (2000j:46109) 
[16] G. Robertson and T. Steger, Asymptotic K-theory for groups acting on $\tilde{A}_{2}$ buildings, Can. J. Math. 53 (2001), 809-833. MR.1848508 (2002f:46141)

[17] W. Szymański, The range of $K$-invariants for $C^{*}$-algebras of infinite graphs, Indiana Univ. Math. J. 51 (2002), 239-249. MR1896162 (2003b:46077)

Department of Mathematics, University of Newcastle, New South Wales 2308, AusTRALIA

E-mail address: stephen.allen@studentmail.newcastle.edu.au

Department of Mathematics, University of Newcastle, New South Wales 2308, AusTRALIA

E-mail address: david.pask@newcastle.edu.au

Department of Mathematics, University of Newcastle, New South Wales 2308, AusTRALIA

E-mail address: aidan.sims@newcastle.edu.au 